\title{
Interactions missionnaires et mariages indiens en zone de frontières (Maragnan début XVIIe siècle)
}

\author{
Charlotte de Castelnau-L’Estoile[1]
}

\begin{abstract}
Résumé
Cette étude analyse la mission en terme d'interaction, enétudiant à la fois la perspective indienne et missionnaire. Indiens et missionnaires sont chacun acteurs dans léchange missionnaire qui n'est jamais éloigné du rapport de force colonial. C'est à travers la question du mariage chrétien des Indiens que linteraction missionnaire est abordée. Le mariage est au centre du projet missionnaire de transformation des Indiens et fait également l'objet de résistance ou de négociation de la part des Indiens. A partir de l'exemple des sources des capucins français du début du XVIIle siècle dans le Maragnan, le texte tente de montrer la grande richesse des écrits missionnaires pour l'histoire et lanthropologie des Indiens en situation de contact avec les missionnaires.
\end{abstract}

Mots-clés: missionnaires; indiens; mariage.

Interações missionárias e matrimônios de índios em zonas de fronteiras (Maranhão, início do século XVIII)

\section{Resumo}

Este estudo analisa a missão em termos de interação, estudando tanto a perspectiva indígena quanto a missionária. Índios e missionários são ambos atores na missão missionária que nunca está distante da relação de poder colonial. É através da questão do matrimônio cristão dos índios que a interação missionária é abordada. O matrimônio é o centro do projeto missionário de transformação dos índios e é da mesma forma o centro de resistência ou de negociação da parte dos índios. A partir do exemplo de fontes de C capuchinhos franceses do início do século XVII, no Maranhão, o texto procura mostrar a grande riqueza dos escritos missionários para a história e a antropologia dos índios em situação de contato com os missionários.

Palavras-chave: missionários; índios; matrimônio.

\section{Interacciones misionarias y matrimonios de indios en zonas de fronteras (Maranhão, inicio del siglo XVII)}

\section{Resumen}

Este estudio analiza la misión en términos de interacción, estudiando tanto la perspectiva indígena como la misionaria. Indios y misionarios son ambos actores en la misión misionaria que nunca está distante de la relación de poder colonial. Es a través de la cuestión del matrimonio cristiano de los indios que la interacción misionaria es abordada. El matrimonio es el centro del proyecto misionario de transformación de los indios y es de la misma forma el centro de resistencia o de negociación de la parte de los indios. A partir del ejemplo de fuentes de capuchinhos franceses del inicio del siglo XVII, en Maranhão, el texto procura mostrar la gran riqueza de los escritos misionarios para la historia y la antropología de los indios en situación de contacto con los misionarios.

Palabras claves: misionarios; indios; matrimonio.

\section{Missionary interactions and Indian marriages in the frontiers (Maranhão early $17^{\text {th }}$ century)}

\section{Abstract}

This study analyzes the mission in terms of interaction, studying as the indigenous perspective as the missionary. Indians and missionaries are actors in the missionary mission that is never far from the colonial power relationship. It is through the issue of the Indians Christian marriage that the missionary interaction is addressed. The marriage is the center of the missionary project of Indians transformation and it is the same as the center of resistance or negotiation for the Indians. From the example of the French Capuchins sources of the early $17^{\text {th }}$ century, in Maranhão, the research shows the wealth of the missionaries writings by the history and the anthropology of the Indian in situation of contact with the missionaries.

Keywords: missionaries; indians; marriage. 
es sources missionnaires ont longtemps été considérées comme contenant un discours univoque sur les progrès de la «conquête spirituelle». Dès lors, selon leurs convictions personnelles, les historiens les ont lues soit pour faire le récit linéaire de l'évangélisation soit au contraire pour dénoncer la dimension de domination d'un tel projet spirituel qui n'apparaissait que comme l'habillage idéologique de la colonisation. Quant aux anthropologues, ils avaient aussi un intérêt pour les sources missionnaires car elles contenaient des informations sur les Indiens, mais ils les lisaient de manière partielle. Ils cherchaient à en extraire l'information sur les Indiens et ne les contextualisaient pas; ils évitaient même de penser la relation missionnaire à l'origine de ces textes car ils étaient uniquement intéressés par les traces d'une culture indigène authentique et originale que les missionnaires auraient consigné dans leurs écrits sans véritablement les comprendre. Depuis une vingtaine d'années, les sources missionnaires font l'objet de nouvelles lectures tant de la part des anthropologues que des historiens. Celles-ci n'apparaissent plus comme le récit du progrès monotone de la conversion, mais elles sont lues parce qu'elles contiennent les traces de l'interaction missionnaire, ce contact prolongé entre Indiens et missionnaires sur fond de rapport de forces, lié à la colonisation. La conversion n'est plus perçue comme une réalité qui s'impose aux Indiens comme une évidence parce qu'elle serait la «Vérité », mais comme un projet de transformation des Indiens que les missionnaires adaptent et reformulent à leur contact. Les Indiens sont désormais analysés comme des acteurs à part entière qui interprètent, s'approprient ou rejettent ce projet que les missionnaires leurs proposent ou tentent de leur imposer selon les circonstances de la colonisation et des rapports de force. De part et d'autre de l'interaction, Indiens et missionnaires s'observent, échangent et négocient. Dans cet échange, la question du contenu des croyances est loin d'être l'unique enjeu, les rituels, les modes de vie (la sédentarisation, la monogamie, le travail) sont au centre des négociations. La mission est ainsi désormais perçue par les historiens comme une interaction entre Indiens et missionnaires que spécialistes d'histoire indigène et d'histoire missionnaire peuvent décrypter. ${ }^{1}$

Cependant, même s'il conçoit la mission comme une interaction, l'historien est confronté d'emblée à la question des sources et à un fort déséquilibre. D'un côté, les missionnaires européens ont laissé des textes de toutes sortes: textes administratifs, règlements, lettres, récits, chroniques. De l'autre, les Indiens n'ont pas écrit, ou très peu. L'historien doit donc ruser, s'attacher à comprendre les réactions indiennes d'après ce qu'en disent les missionnaires, lire entre les lignes et tenter d'écouter, autant que faire se peut, les voix indiennes dans les textes mêmes des colonisateurs. ${ }^{2}$

\footnotetext{
'Pour une présentation plus détaillée de ces enjeux historiographiques autour de l'utilisation par les missionnaires et les anthropologues des sources missionnaires, voir mon article « De l'observation à la conversation: le savoir sur les Indiens du Brésil dans l'œuvre d'Yves d'Évreux », In: Charlotte de Castelnau-L'Estoile; Marie-Lucie Copete; Aliocha Maldavsky; Ines G. Zupanov, Missions d'évangélisation et Circulation des Savoirs XVle-XVIIle siècles, vol. 114, Madrid, Casa de Velazquez, Collection de la Casa de Velázquez, 2011, p. 269-294.

${ }^{2}$ Frank Salomon, « Testimonies: The Making and Reading of Native South American Historical Sources », In: Frank Salomon; Stuart B. Schwartz (eds.), The Cambridge History of the Native Peoples of the Americas, vol. 3, part 1, Cambridge, Cambridge University Press, 1999, chapter 1, p. 19-94.
} 


\section{Indiens tupi et Capucins français au Maragnan au début du XVII ${ }^{\mathrm{e}}$}

Dans cette étude, nous avons privilégié les écrits des missionnaires français, des capucins présents au nord du Brésil au début du XVII siècle. En 1611-1615, des capucins de la province de Paris participent à l'éphémère aventure coloniale de la France équinoxiale dans le Nord du Brésil où les Portugais n'étaient pas encore installés, au Maragnan. ${ }^{3}$ Deux récits de cette aventure coloniale ont été écrits par les capucins Claude d'Abbeville et Yves d'Évreux. Claude d'Abbeville, rentré à Paris, après un séjour au Brésil de six mois en 1612, écrit une Histoire de la mission des Pères capucins en l'isle Maragnan. ${ }^{4}$ Yves d'Évreux, après deux années au Maragnan, écrit une Suitte de l'Histoire des choses plus mémorables advenues en Maragnan les années 1613 \& $1614 .^{5}$

Un des intérêts de ces sources françaises est l'attention exceptionnelle que les deux capucins portent aux paroles indiennes. Claude d'Abbeville insère dans son récit des longues harangues de chefs indiens, comme celles de Iapy Ouassou (chapitre 11) de Momboré Ouassou (chapitre 24). Tout au long de sa chronique, Yves d'Evreux porte lui aussi une grande attention aux voix indiennes. Les six derniers chapitres de son traité sont des « conférences » présentées comme la transcription de longues conversations, que le missionnaire aurait eues avec différents Indiens, chefs ou sorciers. Il ne s'agit pas de croire naïvement que ces conférences sont de simples transcriptions de conversations ou que les harangues indiennes sont des « enregistrements directs ». Il y a évidemment dans ces textes imprimés dans Paris de la seconde décennie du $\mathrm{XVII}{ }^{\mathrm{e}}$ siècle, tout un travail de réécriture, de mise en scène et de mise en forme de la parole indienne. Cependant, pour différentes raisons, qui tiennent tant à la culture des capucins qu'à celle des Indiens tupi, on peut penser que ces voix indiennes, même médiatisées, ne sont pas le pur produit de l'imagination des missionnaires, mais qu'elles comportent une forme de point de vue indien et nous renseignent sur la dimension indienne de l'interaction missionnaire.

Pourquoi une telle attention à la parole indienne dans ces sources françaises? Dans la France du début du XVII ${ }^{\mathrm{e}}$ siècle qui connaît enfin la paix après quarante années de conflits religieux, mais qui reste divisée sur le plan confessionnel, une conférence est un débat contradictoire, écrit ou oral, sur les matières de religion. Ce type de débat a un grand succès à un moment où les armes ont cessé, mais pas « la guerre en papier ", selon l'expression du Mercure Galant de 1605: ${ }^{6}$ libelles, pamphlets poursuivent le combat. Dans ces textes, la parole

\footnotetext{
${ }^{3}$ Pour le contexte, voir Andréa Daher, Les Singularités de la France Equinoxiale. Histoire de la mission des pères capucins au Brésil (1612-1615). Préface de Roger Chartier. Paris, Honoré Champion, 2002.

${ }^{4}$ Claude d'Abbeville, Histoire de la mission des Pères capucins en l'isle Maragnan et terres circonvoisines par le R. P. Claude d'Abbeville, Paris, Imprimerie de Huby, 1614. Disponible sur le site numérique de la Bibliothèque nationale de France, Gallica: <http://gallica.bnffr/ark:/12148/bpt6k57399d>, Accès: mars 2013.

${ }^{5}$ Yves d'Evreux, Voyage au Nord du Brésil, édition F. Denis Paris, 1864. Disponible sur le site numérique de la Bibliothèque nationale de France, Gallica: <http://gallica.bnffr/ark:/12148/bpt6k5732857p>. C'est cette édition que nous utilisons. II existe une nouvelle édition avec le texte complet: Yves d'Evreux, Voyage au Nord du Brésil (1615), édition Franz Obermeier, Kiel Westensee-Verl, 2012.

${ }^{6}$ Bernard Dompnier, Le venin de l'hérésie. Image du protestantisme et combat catholique au XVIle siècle, Paris, le Centurion, 1985, p. 174, cite 166 conférences connues avec précision par des compte-rendus écrits dont 70\% ont été rédigés entre 1593 et 1609.
} 
de l'adversaire religieux est là pour augmenter le prestige de la vérité de l'autre religion. Le public français est donc habitué et est friand de ces joutes oratoires. Il nous semble ainsi que le contexte français de la fin des guerres de religion et de la " tolérance religieuse » explique peut être cette attention à la parole de l'adversaire religieux. A cela s'ajoute le goût d'exotisme du public français. Les Normands qui fréquentent le littoral brésilien depuis le début du XVIe siècle ont souvent ramené des Indiens en France où ils ont toujours fait l'objet d'une grande curiosité. ${ }^{7}$ Claude d'Abbeville et Yves d'Evreux qui écrivent des récits de leur mission en vue de faire connaître l'aventure coloniale de la France équinoxiale et d'amasser des fonds pour rendre possible la suite de cette aventure, abandonnée finalement, répondent en quelque sorte à une demande du public français.

Sur tous les terrains de mission, l'interaction prend souvent une forme de dialogue. ${ }^{8}$ Mais, dans le cas de la société tupinamba, la harangue et le dialogue sont aussi des formes culturelles privilégiées. Chez les Indiens tupi, le prestige des chefs est lié à l'art oratoire à leur capacité à être des « maîtres de la parole » qui est le nom donné aux chefs. ${ }^{9}$ Les belles paroles indiennes contenues dans les textes capucins renvoient à des usages de la société tupinamba.

Pour comprendre et traduire cette parole indienne, les deux pères capucins disposent de traducteurs car ils ne parlent pas bien le tupi même si Yves d'Evreux est resté deux ans au Maragnan s'intéresse de près à la langue indienne Les truchements sont généralement des Français qui ont vécu longtemps au Brésil où ils pratiquaient le commerce du bois brésil et qui ont rejoint l'aventure coloniale de la France équinoxiale. Deux de ces truchements apparaissent dans les textes et sont cités comme étant les interlocuteurs auxquels s'adressent les Indiens, il s'agit du Sieur des Vaux, ${ }^{10}$ Français de Touraine devenu chef de guerre au Brésil sous le surnom de Itajiba (Bras de fer) ou Migan, qui est arrivé enfant au Brésil et qui y a grandi. Pour les chefs indiens, ces truchements sont à la fois des hommes blancs, mais aussi des hommes qui parlent leur langue, qui ont partagé leurs coutumes et noué des alliances avec eux. Ce sont ces hommes qui transmettent et traduisent la parole indienne aux missionnaires.

Ainsi, malgré les réécritures et les mises en forme que suppose la version finale des traités, ces harangues et ces conférences renvoient à des conversations et à des échanges qui eurent sans doute vraiment lieu entre les capucins français et les Indiens du Maragnan. Le sujet principal de ces conversations porte sur le processus

\footnotetext{
'Sur la curiosité française à l'égard des Indiens du Brésil, voir les nombreux travaux de Frank Lestringant, notamment Le Huguenot et le sauvage. La controverse coloniale, en France, au temps des guerres de Religion (1555- 1589), Paris, Klincksieck, 1999.

8Ines G. Zupanov, “I am a great sinner': Jesuit Missionary Dialogues in Southern India (16th century)", Journal of the Economic and Social History of the Orient 55.2-3 (June 2012), Leiden, E. J. Brill, p. 415-446.

${ }^{9}$ Carlos Fausto, "Fragmentos de história e cultura tupinambá. Da etnologia como instrumento crítico de conhecimento etno-histórico," In: Manuela Carneiro da Cunha (org.), História dos Indios no Brasil, São Paulo, Companhia das Letras, 1992.

Sur l'importance du dialogue dans la culture amérindienne, voir Aurore Monod-Becquelin; Philippe Erikson (eds.), Les rituels du dialogue Promenades ethnolinguistiques en terres amérindiennes, Nanterre, Société d'ethnologie, 2000, p. 199-234.

${ }^{10}$ Des Vaux, naturel de Sainte Maure en Touraine, compagnon de Jacques Riffault, est resté longtemps au Brésil; il fait la guerre avec les indiens sous le surnom de Itajiba, bras de fer. II part en France à la demande de ses compagnons demander au roi de France lincorporation du Maragnan à la couronne.
} 
de transformation en cours, sur la christianisation et sur Dieu que l'on désigne sous le nom de Tupan, sur les effets de la présence des pères missionnaires.

Au nord du Brésil, en ce début du XVII ${ }^{\mathrm{e}}$ siècle, les Indiens vivent une période d'incertitudes et de dangers. Les différentes tribus indiennes qui sont installées à cette date dans la région de Maragnan sont depuis longtemps en relation avec le monde des Blancs. Certaines sont de longue date à cet endroit, d'autres sont arrivées plus récemment. Comme le décrit très précisément Fatima Martins Lopes pour la capitainerie du Rio Grande do Norte, il y a un processus d'évasion des Indiens vers les territoires non encore dominés par le pouvoir portugais, le Ceara et au-delà le Maragnan. ${ }^{11}$ Ce processus est ancien, on a trace de migrations pour les capitaineries plus méridionales dès les années 1540; ces migrations se sont intensifiées au fur et à mesure de l'avancée de la colonisation portugaise et de son corollaire, la mise en esclavage des populations indiennes.

«Il nous faut des ferrements, du feu \& des canots

pour lutter contre les Peros (Portugais), et contre les Tapinambos \& autres nations adverses »

Les Français sont également des acteurs importants dans la région, ils commercent avec les tribus du littoral depuis plusieurs décennies. Cette présence s'est encore renforcée après leur expulsion de la baie de Guanabara et de la région de Cabo Frio. En 1561, Jeronimo de Barros, fils du capitaine donataire João de Barros, parle de dix-sept navires français dans la région. De très nombreux liens d'alliance de type matrimonial, de type politique et militaire lient les Indiens du Nord du Brésil, avec les Français. En 1579, une carte dessinée par Jacques de Vau de Claye montre un camp de guerre franco-indien impressionnant où est évoquée la mobilisation possible de 10000 guerriers potiguar (Indiens tupi de la côte du nord) et tapuias (Indiens de l'intérieur des terres (o sertão), mobilisables contre les Portugais. ${ }^{12}$

En 1599, la paix est signée entre les Portugais et les Potiguar. Les capitaineries du Rio Grande do Norte, de la Paraiba sont désormais incluses dans le territoire colonial. La frontière au sens d'espace négocié et encore libre recule plus au nord. A partir des années 1603, le Cearádevient le nouvel espace convoité par les autorités coloniales. Plusieurs expéditions de reconnaissance ont eu lieu comme celle de Pero Coelho en 1603, celles de deux missionnaires jésuites

\footnotetext{
"Fátima Martins Lopes, Índios, colonos e missionários na colonização da capitania do Rio Grande do Norte, Mossoró (RN), Fundação Guimarães Duque, 2003.

${ }^{12}$ Voir le site internet de l'exposition L'Âge d'Or des Cartes marines à la Bibliothèque Nationale de France (2012/2013). La carte de 1579 se trouve à l'adresse suivante: <http://expositions.bnf.fr/marine/grand/por_085.htm>, Accès: septembre 2013.
} 
Francisco Pinto et Luiz Figueira (en 1607-8), de militaires au service du gouverneur, comme Martin Soares de Souza.

Au même moment, les Français prétendent donner une forme coloniale à leur implantation informelle. Le projet de fonder une « colonie » française au Nord du Brésil est ancien mais ce n'est qu'en 1612 que l'expédition de la France équinoxiale s'installe. Les Français viennent au nom de la reine de France, Marie de Médicis, prendre possession de la terre avec un projet colonial, des missionnaires, des règlements. La colonie est fragile, c'est encore un projet qui dépend de ce qui arrivera dans les prochains bateaux. Elle n'a pas eu vraiment le temps de se déployer: les Français, abandonnés par leur monarchie qui renonce à son projet d'expansion coloniale, sont facilement délogés par les Portugais appuyés par la monarchie de l'Union des couronnes qui désormais envisage la conquête du Nord du Brésil et de l'Amazonie. La ville de Belém est fondée en 1615.

Pendant la courte période française, la situation des Indiens est caractérisée par une grande incertitude, et par la recherche de stratégies d'adaptation. Ils connaissent le projet de domination des Portugais et l'esclavage, ils comprennent aussi que les Français ne sont plus dans une phase d'échanges et de troc. On trouve ainsi, entre les lignes de ces récits missionnaires, une histoire des Indiens dans la colonie à un moment critique: celui du contact colonial et de l'entrée dans le monde dominateur des Blancs et c'est le sujet principal des conversations et des harangues. A partir de ces échanges entre le missionnaire et les Indiens, l'historien perçoit l'évangélisation comme un processus négocié de transformation des Indiens confrontés à une situation coloniale qu'ils ne dominent pas.

La Conférence avec Jacoupen, rapporté dans le livre d'Yves d'Evreux, au Chapitre XIX, ${ }^{13}$ est un bel exemple de conversion en situation coloniale. Dans les propos de ce principal de la tribu des «Canibaliers ", qui demande à Yves d'Évreux le baptême, il apparaît clairement que le chef indien a le projet d'acquérir la force dispensée par Tupan le Dieu des Blancs et notamment les armes des Français. Voici comment il s'adresse au missionnaire: «Il m’ennuye fort que je ne suis baptisé: car je recongnais que tandis que je demeureray demeurerai comme je suis, le Diable me peut travailler \& donner de la peine ».

Il fait, ensuite, le récit des malheurs que vient de connaître son peuple. Originaire de Pernambouc, sa tribu a entrepris une migration, conduite par un chamane: « afin d'aller posséder une belle terre, en laquelle naturellement toutes choses viendraient à souhait, sans qu'ils n'eussent aucune peine ny travail ».

Au cours de cette migration, les hommes périrent par milliers. Les survivants furent recueillis par le français La Ravardière, et amenés à l'Ile de Maragnan par bateau. Là, ils engagèrent un combat contre une autre tribu, les Tapinambos, où plus d'une centaine d'homme furent tués. Iacoupen conclut sa triste histoire: «Il nous faut des ferrements, du feu \& des canots pour lutter contre les Peros (Portugais), et contre les Tapinambos \& autres nations adverses ».

A la suite de cette première conversation, Yves nele juge pas encore prêtà recevoir le baptême, il le renvoie chez lui en lui disant d'apprendre « les merveilles de Tupan ».

${ }^{13} Y$ ves d’Evreux, Voyage au Nord du Brésil, Paris, Édition F. Denis, 1864, p. 348-354. 
Iacoupen revient pour discuter avec Yves d'Évreux de « la diversité des Nations »:

Je voy que les Français abondent en richesses, sont valeureux, ont inventé les Navire pour passer les Mers, les canons \& la poudre, pour tuer les hommes invisiblement [...] Et au contraire tous nous autres de par-deçà nous sommes demeurez errans \& vagabons, sans habits, sans haches, serpes, couteaux \& autres ferrements.

Dans cette conversation rapportée par Yves d'Évreux et qui met habilement en valeur la supériorité des Blancs et de leur Dieu sur les Indiens, c'est cependant Jacoupen qui a l'initiative: il se déplace, demande le baptême, choisit les sujets de conversation. Il entraîne le missionnaire à discuter des armes et de la puissance que Dieu accorde à ceux qui le reconnaissent. Yves d'Évreux ne condamne pas l'Indien pour sa vision « intéressée » de la conversion, il semble au contraire utiliser la motivation du principal pour l'amener à approfondir sa connaissance de Dieu. Il ne le baptise pas car il réserve le sacrement, sauf exception, aux enfants ou aux mourants. Le missionnaire évoque néanmoins à propos de Jacoupen, «la capacité de ces âmes à recevoir la Foy » et lui fait une réponse vague, qui est à la fois conforme à la doctrine chrétienne mais aussi aux aspirations du principal: « Et quand Dieu voit qu'un homme est disposé à recevoir sa Foy, il ne manque point de le visiter par ses Apôtres, lesquels luy donnent le moyen de se sauver. »

La conversion au christianisme est présentée, dans le dialogue entre le missionnaire et l'indien, comme une réponse adaptée au nouveau contexte colonial, une solution aux difficultés du temps.

On peut parler ici de religion coloniale, au sens de réponse culturelle forgée par les Indiens et les missionnaires, en réponse à une situation donnée, celle du contact colonial. Les capucins professent au Maragnan une religion adaptée à la demande indienne: une religion du Dieu tout-puissant, Tupan, victorieux du Diable Giropary et présentée comme la seule alternative possible pour les Indiens, confrontés à la colonisation des Portugais et des Français. La religion n'est pas seulement transmise par les missionnaires aux Indiens, elle devient une forme de médiation culturelle, un langage réélaboré par les cultures indigènes à partir de leurs propres représentations et adapté aux nouvelles nécessités du contexte colonial. ${ }^{14}$

L'adoption de la « civilisation » et du christianisme signifie ainsi une transformation profonde du mode de vie, et passe par un bouleversement des rapports familiaux. Les Indiens négocient, avec le missionnaire, ce passage au modèle chrétien. Dans ces conversations entre Indiens et missionnaires, la question du mariage apparaît comme l'une des conséquences les plus fondamentales du processus de colonisation et d'évangélisation. C'est autour de la question du mariage que je me propose d'analyser l'interaction missionnaire dans ce présent article. ${ }^{15}$ Que signifiait pour les Indiens et pour les missionnaires le mariage chrétien? Quel en étaitl'enjeu?

14Jean Comaroff; John Comaroff, Of revelation and revolution. Christianity, Colonialisme and Consciousness in Southe Africa, Chicago, University of Chicago Press, 1991. Pour le Brésil colonial, Cristina Pompa a présenté ce type d'interprétation dans Religião como tradução Missionários, Tupi e Tapuia no Brasil colonial, Bauru (SP), Edusc, 2003. ${ }^{15}$ Cet article s'intègre dans mes recherches actuelles sur le mariage des Indiens et des esclaves dans le Brésil colonial, Les chaînes du mariage, Catholicisme, Colonisation et Esclavage, Brésil colonial XVI-XVIII siècle, ouvrage inédit présenté pour le dossier d'habilitation à diriger des recherches, Paris Sorbonne, juin 2013. 


\section{Le mariage, enjeu essentiel de l'interaction entre missionnaires et Indiens}

\section{La vision indienne du mariage chrétien}

Dans le récit de Claude d'Abbeville, c'est insérée une belle harangue de Momboré Ouassou, un vieux chef potiguar du nord du Brésil qui s'adresse aux hommes blancs venus planter une croix dans son village pour marquer l'alliance de sa tribu avec la France équinoxiale. Âgé de 90 ans, originaire de la région du Pernambouc, Momboré Ouassou a donc une longue expérience des échanges avec les Européens et du processus de colonisation. Son discours fait l'historique de la présence des Portugais qu'il nomme les Pero (ce qui signifie chiens): des premières relations de troc jusqu'aux relations d'esclavage. Il évoque ses craintes que désormais les Français cherchent à agir de même et veulent remplacer les relations d'alliance par une domination. Pour Momboré, la croix et la venue des Pay (les prêtres) sont des signes inquiétants. Le mariage occupe une place centrale dans le raisonnement du vieil Indien. Écoutons-le:

J'ai vu (ce dit-il) l'établissement des Pero, à Fernambourg \& à Potyio, lesquels ont commencé tout ainsi que Vous autres François, vous faites maintenant. Au commencement, les Pero ne faisoient que traffiquer avec ceux de ces lieux là, sans se vouloir autrement habituer: Et pour lors, ils couchaient librement avec leurs filles; ce que nos Semblables de Fernambourg \& à Potyiou, tenoient à grand honneur. [...]

depuis ils leur firent entendre, qu'ils ne pouvoient prendre leurs filles en cette sorte, que Dieu leur deffendoit de s'en servir sinon par mariage, \& aussi qu'ils ne devoient se marier avec elles, si elles n'estoient Baptisés, pour ce faire, il estoit nécessaire d'avoir des Pay. Davantage ils leur persuadèrent si bien qu'ils ne pouvaient se passer d'esclaves (ni les Pay aussi) pour faire leur mesnage \& travailler pour eux, qu'on fut contraint de leur en donner. [...]

De même Vous autres François, quand au commencement vous veniez en ce pays, cen'était que pour traffiquer simplement avec nous, aussi ne faisiez vous pas de difficulté, non plus que les Pero de coucher avec nos filles, \& nous nous estimions bienheureux quand elles en pouvaient avoir des enfans. [...]

Depuis que les Pay font venus, Vous avez planté des Croix, ainsi queles Pero, Vous commencez à instruire \& baptiser, ainsi que les Pero:vous dites que vous ne pouvez vous servir de nos filles, sinon en mariage \& quand elles auront receu le baptesme, comme disaient les Pero. Au commencement Vous ne vouliez pas d'esclaves, non plus que les Pero, maintenant vous en demandez \& vous en voulez avoir comme ils firent en la fin. Je ne crois pas pourtant que Vous avez le même dessein que les Pero, aussi n'en ay-ie pas de crainte: car étant vieil comme je suis, desormais je ne crains plus rien: mais enfin je dis ingénument que ce que j'ai vu de mes yeux. ${ }^{16}$

\footnotetext{
${ }^{16}$ Claude d'Abbeville, Histoire de la mission des Pères capucins en lissle Maragnan et terres circonvoisines par le R. P. Claude d'Abbeville, Paris, Imprimerie de Huby, 1614. Disponible sur le site numérique de la Bibliothèque nationale de France, Gallica: <http://gallica.bnffr/ark:/12148/bpt6k57399d>, Accès : mars 2013. Chapitre XXIIII: De ce qui se passa à Eussaouap pendant notre visite. Remontrance du vieux chef Momboré Ouassou, p. 148-150.
} 
Momboré propose un enchaînement implacable des faits à partir de l'expérience de la colonisation portugaise. Au départ, les Portugais tout comme les Français ont été dans une relation de «trafic », d'échange commercial avec les Indiens. Ils n'étaient que de passage sur la terre brésilienne. Ils couchaient librement avec les filles des Indiens et les Indiens en tiraient un grand honneur. Momboré évoque de manière implicite les règles des unions indiennes. Ce sont les pères qui offrent les filles à des hommes qui deviennent leurs gendres. Ceux-ci doivent un service à leur beau père, soit d'ordre symbolique soit matériel. L'arrivée des Européens signifie pour les Indiens des possibilités nouvelles d'alliance et de profit. Les pères offrent très volontiers leurs filles à ces hommes. La logique des unions indiennes et l'insertion des Européens dans le système d'alliance et d'échange des femmes sont ainsi clairement expliquées par Momboré.

\section{Du point de vue des missionnaires, il s'agit là d'un accident, en quelque sorte, car cette femme était censée mourir après avoir reçu le baptême}

Dans un second temps, les Portugais se sont installés dans une forteresse. Les hommes n'ont plus voulu des femmes que les Indiens continuaient à leur proposer au prétexte que Dieu le leur interdisait sauf en mariage et ils ont fait venir les prêtres qui ont parlé de baptême et ont planté des croix. Le mariage chrétien s'est imposé. Les Européens exigent que le mariage chrétien devienne la règle. Enfin, le troisième temps est celui de l'esclavage, les Portugais mettent en esclavage non seulement des prisonniers faits à la guerre mais des enfants et donc de la nation entière. Pour les Indiens, c'est le temps de la fuite. Momboré ne parle plus de mariage dans ce troisième temps.

C'estfinalement sa propre vie que raconte Momboré. Originaire du Pernambouc, né vers 1520, le vieillard a connu les premières relations de trafic du bois brasil durant son enfance, quand les Portugais se contentaient de passer prendre le bois de teinture amassé sur le rivage par les Indiens et l'échangeaient contre des hameçons, des haches et d'autres objets très prisés. L'installation à demeure des Portugais s'est faite en deux temps, à partir de 1530 avec la création d'une feitoria (comptoir portugais) pour le commerce du bois brasil dont la couronne avait le monopole, puis la fondation de la capitainerie en 1534 avec l'installation du capitaine donataire Duarte Coelho et de sa femme Beatriz de Albuquerque, tous deux issus de familles de la noblesse du Portugal. L'exceptionnel succès de la capitainerie du Pernambouc repose justement sur l'alliance avec les Indiens. Le beau-frère du capitaine, Jeronimo de Albuquerque symbolise cette alliance: il a une longue relation avec la fille de Arcoverde, le chef des Indiens Potiguar de la région. Momboré quand il évoque le grand honneur ressenti par ces alliances entre Portugais et Indiennes, fait peut-être allusion à cette union en particulier, qui est un exemple d'union entre la noblesse du Portugal et celle de la terre. 
Fort de cette alliance avec les Indiens, Duarte Coelho introduit la culture de la canne à sucre dans sa capitainerie. C'est avec l'aide des Indiens de la région qu'il défriche et construit les cinq moulins qui sont en état de fonctionnement en 1550. Seules parmi les autres capitaineries du Brésil, Pernambouc et São Vicente ont réussi une première colonisation et mise en valeur; toutes deux s'appuient sur l'alliance avec les Indiens. ${ }^{17}$ A São Vicente, le personnage clef de l'alliance, l'intermédiaire essentiel, est João Ramalho, portugais installé depuis 1511 au Brésil, gendre de Tiribiça, chef tamoio de Piratininga. ${ }^{18}$ Dans les deux cas, l'alliance entre Portugais et Indiens est scellée par ces filles données par leurs pères aux étrangers.

A Pernambouc, la relation entre les Portugais et les Indiens se détériore avec l'arrivée de nombreux colons, souvent des exilés qui arrivent du Portugal (degredados) et qui veulent toujours plus d'esclaves. Duarte Coelho écrit au roi pour se plaindre de ces hommes: «nous avons perdu la confiance que jusqu'à maintenant les Indiens avaient à notre égard ». Cela signifie que les nouveaux venus ne respectent pas les alliances avec les Indiens et ne se contentent pas des ennemis captifs comme esclaves. C'est également au début des années 1550 qu'ils arrivent à Pernambouc les premiers jésuites, qui remarquent dans leur correspondance, le grand nombre d'esclaves et qui cherchent à l'imposer le mariage chrétien à cette société mélangée. Pour Momboré, esclavage, mariage et conversion vont de pair et font leur apparition au milieu du XVI siècle. A partir de 1560, la demande incessante d'esclaves provoque une guerre générale et la fuite des Indiens vers le nord. Les sources jésuites et coloniales corroborent les souvenirs du vieillard indien et son schéma narratif est donc exact. A l'échelle d'une longue vie, cet Indien a vu se dérouler tout le processus colonial de l'alliance et l'échange jusqu'à l'esclavage et la guerre. Dans ce récit, le passage des alliances indiennes au mariage dans les termes chrétiens est un moment clef de l'imposition de la domination: le refus des filles indiennes de la part des Européens équivaut au renoncement à l'échange à parts égales. De même, dans le récit, l'imposition du mariage chrétien et l'esclavage se suivent de peu. Le discours de Momboré provoqua l'émoi des Indiens qui le trouvèrent sans doute très véridique. Les Français durent tenter de persuader les Indiens que leurs intentions n'étaient pas celles des Portugais. Cette version «indienne» de l'histoire de la colonisation du Brésil au $\mathrm{XVI}^{\mathrm{e}}$ siècle fait donc la part belle au transfert du mariage chrétien comme un moment essentiel dans l'imposition de la domination européenne.

\section{Le mariage chrétien selon les missionnaires}

Avant le discours de Momboré Ouassou, un autre chef indien Iapy Ouassou s'était interrogé sur le mariage et sa harangue se trouve au chapitre $11 \mathrm{du}$ récit de Claude d'Abbeville. Le missionnaire Yves d'Evreux avait répondu aux

\footnotetext{
${ }_{17}$ Maria Regina Celestino de Almeida, Os indios na História do Brasil, Rio de Janeiro, FGV, 2010, chap. 1. ${ }^{18}$ On peut utiliser la notion d'intermédiaire « go between » entre le monde indien et le monde européen développé, entre autres, par Alida Metcalf pour la colonisation du Brésil. Alida G. Metcalf, Go-betweens and the Colonization of Brazil 1500-1600, Austin, TX, University of Texas Press, 2005.
} 
interrogations du chef indien. Dans un premier temps, le missionnaire disqualifie les alliances que les Français avaient coutume de contracter avec les Indiennes comme de la "prostitution »: les Français acceptent d'entrer dans les relations d'alliance entre beau-père et gendre et offrent des cadeaux (hameçons, haches) aux hommes en échange des femmes. Ensuite, le père capucin explique aussi le mariage des chrétiens, mettant en valeur sa dimension religieuse.

Pour ce qui est des femmes, Dieu nous commande, à nous autres, de ne nous marier jamais, \& nous desfend absolument leur compagnie, afin de le servir plus purement; ne voulant pas que ses sacremens soient maniez que par ceux qui vivent en continuelle chasteté. Quant aux autres chrestiens qui sont ses enfans par le moyen du baptisme, il les laisse libre de se marier s'ils veulent, \& leur permet d'avoir une femme seulement \& non davantage, comme aux filles d'avoir un seul mari sans jamais se pouvoir quitter: que s'ils se quittent il ne veut pas qu'ils en prennent d'autres: car les hommes qui ont des plusieurs femmes, \& les femmes ou filles qui s'abandonnent à plusieurs maris, se sont pas vrais enfans du grand Toupan, mais serfs de Ieropary qui est le Diable.

Que si quelqu'un d'entre vous desire d'être enfant de Toupan, $\&$ recevoir le saint Baptisme; il faut qu'il se résoudre à quitter la pluralité des femmes qui se permet entre vous, c'est à vous à y aviser. Il ne nous importe aucunement: nous ne sommes pas ici venus pour vous contraindre à quoi que ce soit, mais pour vous enseigner (avec le plus de douceur qu'il nous sera possible) quel est le vrai Toupan \& comme il faut servir et adorer.

Si les François refusent vos filles ce n'est pas nous qui les empêchent, trop bien les prions nous de se ressouvenir qu'ils sont enfans du grand Toupan, qui leur prohibe d'en abuser, \& comme tels ne doivent pas désobéir à ce qu'il leur commande. Aussi est-ce une chose bien deshonneste à vous autres de prostituer ainsi vos filles, \& à elles de se donner à tous venans comme elles font, vous montrez bien par là que vous êtes enfans de Ieropary: Si donc vous désirez d'eviter les tourmens qu'il vous prépare, il faut nécessairement quitter toutes ces damnables coutumes, \& se ranger à celles des vrais enfants de Toupan. ${ }^{19}$

Le missionnaire expose ainsi la doctrine chrétienne du mariage au public indien. Le mariage doit être monogame et indissoluble. Comme le baptême, il est un sacrement, c'est-à-dire un moyen d'être enfant du grand Tupan. La mention de Tupan (Dieu en langue générale) et de Giropary (le Diable) montre bien que le missionnaire cherche à faire comprendre aux Indiens que c'est leur salut qui est en jeu. Selon le missionnaire, les coutumes qui mènent les Indiens à l'enfer sont la pluralité des femmes et les alliances multiples. Le choix de la conversion implique d'adopter la monogamie.

\footnotetext{
${ }^{19}$ Claude d'Abbeville, Histoire de la mission des Pères capucins en lisle Maragnan et terres circonvoisines par le R. P. Claude d'Abbeville, Paris, Imprimerie de Huby, 1614. Disponible sur le site numérique de la Bibliothèque nationale de France, Gallica: <http://gallica.bnffr/ark:/12148/bpt6k57399d>, Accès : mars 2013., Chapitre XI: Discours notable de Japy Ouassou principal de lissle de Maragnan, \& de quelques questions remarquables quili nous fit, p. 74v-75rv.
} 
La harangue du chef indien et l'exposé doctrinal du missionnaire tels que Claude d'Abbeville les donne à entendre dans sa chronique, sont des indices que le mariage devait constituer un élément important de l'interaction missionnaire, un thème central des discussions entre les pères et les Indiens, un sujet d'incompréhension de part et d'autre.

\section{L'objectif missionnaire: la transformation des alliances indiennes en mariage chrétien}

Alliance, prostitution, mariage, conversion, colonisation et esclavage, tels sont les termes employés lors de ces échanges entre missionnaires et Indiens au sujet des unions conjugales. Certes, les missionnaires exposent la doctrine chrétienne du mariage aux Indiens comme ils le font pour les autres thèmes du catéchisme; mais dans le cas particulier du mariage, ils ne font pas qu'enseigner et instruire ce qu'ils considèrent comme la "vérité », ils observent aussi et tentent de comprendre les pratiques indiennes des unions conjugales. Pourquoi cette attention à l'autre sur ce point précis?

\section{Mais contrairement à ceux qui sont restés en surface, le missionnaire a ensuite cherché à comprendre ( "pénétrer et remarquer ») les règles de «la nature», dans ces gens « dénués de grâce »}

En fait, il ne s'agit ni d'une curiosité gratuite ni d'un intérêt savant particulier pour la famille, cette attention aux mariages indiens vient de la volonté missionnaire d'introduire le mariage chrétien chez les Indiens. En effet, dans la doctrine chrétienne du mariage, il est établi que le mariage selon la loi naturelle existe chez tous les hommes. Ce mariage naturel est un engagement à mener une vie commune, à avoir des enfants. Les théologiens pensent aussi que les règles des mariages varient selon les différentes sociétés qui, pour la plupart, acceptent la polygamie ou la répudiation et le remariage. Le mariage chrétien serait, selon eux, la forme achevée et parfaite du mariage, un mariage indissoluble et monogame.

L'Église qui est née dans l'empire romain, mosaïque de peuples divers, a, dès ses origines, pensé la question des mariages des infidèles et des convertis. En principe, les missionnaires ne procèdent pas à de nouveaux mariages puisqu'ils pensent que les mariages infidèles sont de vrais mariages, quand des infidèles se convertissent, leur mariage est transformé en mariage chrétien par une simple bénédiction. En cas de polygamie, la première union est considérée comme le seul vrai mariage. Telle est la 
doctrine chrétienne de transformation des mariages indiens en mariages chrétiens et ce sont ces règles qui expliquent l'intérêt des missionnaires pour les coutumes conjugales et familiales des Indiens. ${ }^{20}$

\section{L'observation des coutumes indiennes}

Dans son traité des coutumes indiennes, Yves d'Évreux consacre ainsi plusieurs chapitres à la famille indienne. Les chapitres 21 et 22 décrivent les différents âges de la vie chez les hommes et les femmes et s'intitulent « Ordre et respect que la Nature a mise entre les sauvages... », le chapitre 23 porte sur la consanguinité. Il a entrepris une véritable enquête, ne se contentant pas de répéter les autres auteurs français sur le sujet. Il traite, notamment, en détail de la couvade des hommes qui n'avait pas été décrite aussi précisément par les autres sources. ${ }^{21} \mathrm{Il}$ signale le lien entre guerre et mariage: c'est au moment où les hommes sont «bons guerriers pour bien frapper » qu'ils recherchent les femmes en mariage. La polygamie était, de fait, réservée aux guerriers valeureux qui avaient tué des ennemis à la guerre, et le premier mariage avait lieu, pour les hommes, après la mort de leur premier ennemi.

La dimension économique et sociale de la polygamie est évoquée par le capucin, qui consacre une longue analyse à la place des femmes dans la société tupi. Ce sont « elles [qui] ont la charge nécessaire pour le vivre de la famille »: elles jardinent, tissent, cuisinent et « comme les mulets de par-deçà » portent les bagages, occupation très importante dans une société semi-nomade. Yves d'Évreux en conclut que «Ces Sauvages sont extrêmement convoiteux d'avoir nombre de femmes », pour le prestige que cela donne: «ils sont prisés et estimés selon le nombre de femmes qu'ils ont ». Yves d'Évreux remarque qu'il n'y a pas de dot, ni de douaire au moment du mariage, mais un engagement de la part du gendre à subvenir aux besoins de son beau-père. Pour le prouver, le missionnaire transcrit au style direct des extraits de conversations entre époux. Il donne les termes pour désigner chaque âge de la vie ainsi que tous les termes de parenté, avec une très grande précision, en distinguant parentèle matrilinéaire et patrilinéaire (la seule qui compte pour les interdits de consanguinité), descendance légitime et bâtardise (qui concerne les unions avec les prisonniers de guerre). Toutes ces données, recueillies par le missionnaire, ont été utilisées par les anthropologues des sociétés tupi, comme Florestan Fernandes qui s'est appuyé sur la description d'Yves d'Evreux pour bâtir sa théorie de la gérontocratie tupi. ${ }^{22}$

Cependant, les informations sur la famille indienne se comprennent mieux si l'on s'intéresse à la dimension missionnaire du texte. Yves d'Évreux n'a pas

\footnotetext{
${ }^{20}$ Charlotte de Castelnau L’Estoile, «Le mariage des infidèles au XV|e siècle: doutes missionnaires et autorité pontificale», Mélanges de l'Ecole Française de Rome Italie-Méditerranée, 2009, n. 1, 2009, p. 95-121.

${ }^{21} Y$ ves d'Evreux, Voyage au Nord du Brésil, Paris, Édition F. Denis, 1864, p. 89: «il se couche pour faire la gesine au lieu de sa femme, qui s'employe à son office coutumier, \& lors toutes les femmes du village viennent le voir, le consolant sur la peine $\&$ la douleur qu'il a eu de faire cet enfant ».

${ }^{22}$ Florestan Fernandes expose sa théorie de la gérontocratie au chapitre III « O sistema de parentesco », In: _.., A organização social dos Tupinambá, UnB, São Paulo, 1989 [1948].
} 
simplement observé les coutumes indiennes, il les a observées et interprétées selon sa grille missionnaire de transformation des mariages des Indiens. Parce qu'il est un missionnaire et qu'il a pour ambition de transformer le mariage des Indiens, le père Yves s'attache à comprendre en profondeur les règles qui gèrent la vie familiale des Indiens. ${ }^{23}$ Il explique lui-même l'acuité de son regard sur cette question, en se comparant aux autres observateurs:

combien y en a-il, qui ont ignoré, \& ignorent ce que j'ai rapporté ici, \& rapporterai, quoi qu'ils aient longtemps conversé avec eux, faute d'avoir pénétré et remarqué la belle conduite de la nature en ces gens destitués de grâce, mais ont passé par dessus ces pierres précieuses sans en faire leur profit, traversant le tout en gros. ${ }^{24}$

Il y a, dans ce passage sur le mariage, une sorte d'autodéfinition du regard du missionnaire sur les Indiens. Comme ceux qu'il qualifie d'ignorants et qui sont, par opposition à lui, les voyageurs ou les truchements, le missionnaire a " conversé » pour obtenir des informations. Le choix du verbe « converser » est intéressant et se démarque du verbe « observer», souvent choisi pour qualifier le travail de description des coutumes. Les termes de la parenté, qui émaillent toute la description d'Yves d'Évreux, renvoient à ces conversations en tupi, origine de son savoir. Le savoir du missionnaire consiste à répéter ce que les Indiens lui ont dit, de leur organisation familiale.

Mais contrairement à ceux qui sont restés en surface, le missionnaire a ensuite cherché à comprendre ( "pénétrer et remarquer ») les règles de "la nature », dans ces gens « dénués de grâce ». Yves d'Évreux explique, ici, de manière elliptique le travail du missionnaire, cherchant évaluer les unions indigènes à l'aune de la loi naturelle. La formule qu'il emploie revient à dire que les unions des Indiens tupi, qui ne sont pas conformes aux lois du christianisme (la loi de Grâce), sont, cependant, conformes à la loi naturelle. Selon l'interprétation du missionnaire, Dieu a imprimé la loi naturelle dans tous les hommes, et « un ordre et un respect » naturels sont perceptibles dans les règles d'unions des Indiens tupi. Les chrétiens devraient même s'en inspirer: la gratitude pour les anciens, le travail du gendre pour le beau-père sont pour lui exemplaires. ${ }^{25}$ Le missionnaire commente à partir de là le « tu quitteras ton père et ta mère » en suggérant que tous les enfants de la chrétienté apprennent des Indiens « la vraie intelligence de ces paroles formelles du mariage », par lesquelles Dieu n'a pas voulu signifier que le mariage signifiait l'ingratitude envers les parents, mais seulement l'union de l'homme et la femme. La formule est frappante. Elle signifie que les Indiens tupinamba, polygames et qui n'ont pas une vision morale de la sexualité (à plusieurs reprises le capucin évoque sa " pudeur », pour signaler qu'il n'entre pas plus avant dans la description), sont, malgré tout, exemplaires sur le mariage. Il y a

\footnotetext{
${ }^{23}$ De la même façon, en Nouvelle Calédonie au XXe siècle, la connaissance de la famille kanak par Leenhardt peut s'expliquer par la préoccupation du pasteur protestant pour le mariage. Michel Naepels; Christine Salomon (eds.), Terrains et destins de Maurice Leenhardt, Paris, Éditions de l'École des Hautes Études en Sciences Sociales, 2007.

${ }^{24}$ Yves d'Evreux, Voyage au Nord du Brésil, Paris, Édition F. Denis, 1864, p. 86.

25/dem, Ibidem, p. 83.
} 
là une vraie différence de jugement par rapport aux jésuites du XVI ${ }^{\mathrm{e}}$ siècle qui estimaient qu'il n'y avait pas de mariage naturel dans la société tupi car pas de sens de l'obligation entre les époux tupi qui pouvaient se séparer pour le motifle plus futile qui soit sans que les conjoints délaissés n'en ressentent d'amertume.

Pour Yves d'Evreux, il y a un vrai mariage (selon la loi naturelle) chez les Indiens tupi et toute sa description de la famille tupi est empreinte de ce jugement sous-jacent. Cet exemple précis montre qu'une lecture décontextualisée des sources missionnaires pour en extraire seulement des informations sur les Indiens que les missionnaires auraient consigné sans les comprendre, risque d'être appauvrissante. C'est au contraire en comprenant au plus près les enjeux de l'interaction missionnaire que l'on peut au mieux interpréter ces sources. En négligeant le point de vue missionnaire qui était de rechercher des équivalences entre unions indiennes et mariage chrétien, les anthropologues ont lu les textes de description des coutumes conjugales indiennes de manière trop littérale. Pour en revenir à l'exemple de Florestan Fernandes, le sociologue reprend sous forme de catalogue les coutumes consignées par les missionnaires en ignorant le grand débat autour de l'existence ou non d'un mariage naturel chez les Indiens du Brésil mais il reconnaît lui même des formes de contradictions dans ces sources: Dans sa partie intitulée Regras relativas ao matrimônio e à organização da família, il souligne par deux fois l'importance du mariage: «le mariage constituait une institution de grande importance dans le système socio culturel Tupinamba. ${ }^{26}$ L'absence de contextualisation de la production des sources missionnaires fait qu'il y relève des informations contradictoires sans tenter d'expliquer la raison de ces contradictions. Il évoque la difficulté à y voir clair dans les règles: y a-t-il une épouse préférentielle ou pas? y a-t-il toujours pluralité des épouses? Il parle de «monogamie occasionnelle et polygamie occasionnelle » qui reflète bien cette incertitude et il conclut sur un sentiment de ne pas tout comprendre:

\section{Tenho a impressão, no entanto baseado nas varias possibilidades de escolha do cônjuge, e de fixação da residência que uma ampla esfera do sistema de parentesco Tupinambá permanece desconhecida. ${ }^{27}$}

Il est intéressant de constater que le sociologue, travaillant avec des sources missionnaires abondantes dont il n'interroge pas les fondements (Pourquoi tant de descriptions? Dans quel objectif?), aboutit au constat que le système de parenté tupi reste inconnu. En fait, les sources missionnaires reflètent elles-mêmes la perplexité des auteurs ou leurs prises de position contradictoires sur le débat de la nature véritable des unions conjugales indiennes.

\footnotetext{
${ }^{26}$ Florestan Fernandes, A organização social dos Tupinambá, UnB, São Paulo, 1989 [1948], p. 183-212. 27"J'ai l'impression, à partir du fait qu'il existe diverses possibilités de choix du conjoint ou de la fixation de la résidence, qu'une grande partie du système de parenté Tupinamba demeure méconnue."(TN). Florestan Fernandes, A organização social dos Tupinambá, UnB, São Paulo, 1989 [1948], p. 183-212.
} 


\title{
Négociations sur le mariage
}

La chronique d'Yves d'Évreux contient non seulement une riche description du système indigène de parenté et d'alliance, mais il montre ce système en voie de transformation, du fait de la présence des missionnaires. La deuxième partie de la chronique consiste en un " traité spirituel », un récit du processus d'évangélisation. Le thème du mariage est au centre des conversations des Indiens avec le missionnaire, rapportées dans ce traité spirituel. Yves explique, au chapitre trois du traité spirituel, que les missionnaires demandaient aux adultes deux choses pour les baptiser: savoir la doctrine chrétienne et renoncer à la pluralité des femmes. Se convertir au christianisme revenait très concrètement à abandonner la polygamie: une démarche sans aucun doute coûteuse pour les Indiens.

Dans le premier chapitre, où Yves d'Évreux montre le grand enthousiasme des Indiens pour la nouvelle religion, l'abandon de la polygamie ne semble pas poser de difficultés. En effet, les principaux disent même vouloir épouser des femmes de France et n'en vouloir qu'une: «j'aime une femme françoise de tout mon cœur, je l'aime extremement ». Cependant, le désir d'épouser une femme de France, fort signe de prestige et promesse de richesses, est une forme d'utopie missionnaire, comme s'il s'agissait de rendre la monogamie plus attirante ou de l'inscrire dans un avenir lointain et hypothétique, car les femmes de France ne sont pas encore là.

Dans les cas concrets et individuels, on sent que le changement de situation matrimoniale est délicat et fait l'objet de tractations. Ainsi, Yves d'Évreux commence par dire à Pacamont, le grand «barbier» (sorcier) de Comma qui est arrivé, juché sur le dos de sa plus forte femme, et qui veut « pouvoir parler avec Toupan », qu'il doit abandonner ses trente femmes. Pacamont s'en retourne, hésitant. ${ }^{28}$

Le missionnaire a une discussion sur la pluralité des femmes, avec Martin, un principal de Tapouytapere:

\begin{abstract}
Il me répliqua, que pour la pluralité des femmes, c'etait chose qu'il n'avait jamais guères approuvée, \& qu'il etait plus raisonnable qu'un homme n'eust qu'une seule femme mais que pour le bien de son menage, il en avait besoin de plusieurs. Illui dit là dessus qu'il pouvait avoir des plusieurs femmes en qualité de servantes, mais non en qualité de femmes. A quoi il s'accorda facilement.
\end{abstract}

Un peu plus loin, Yves revient sur le règlement de la situation matrimoniale de Martim:

Il nous promit semblablement qu'il élisait une de ses trois femmes, spécialement celle qui étaitmère de cetenfant, si tantétait qu'ellevoulut se faire Chrestienne comme lui: pour deux autres, qu'il les retiendroit comme servantes. Il s'est fort bien acquitté de ses promesses. ${ }^{29}$

Martin est un modèle de nouveau chrétien, il est le seul adulte, non malade, qui reçoit le baptême dans le récit d'Yves d'Évreux. Il a apparemment réglé sa

\footnotetext{
${ }^{28}$ Yves d'Evreux, Voyage au Nord du Brésil, Paris, Édition F. Denis, 1864, Second traité, chapitre 16. 29/dem, Ibidem, chapitre 3.
} 
situation matrimoniale sans difficulté. L'insistance du texte, qui revient deux fois sur l'épisode, souligne que cette condition était absolument nécessaire; le cas de Martin est donc exceptionnel et non généralisable.

La conversion des unions indiennes en mariages chrétiens, est un authentique travail pour les missionnaires de terrain. Yves d'Evreux expose, à travers le cas du baptême d'une femme de la tribu Tobajara, les difficultés posées par la transformation des unions indigènes en mariage chrétien..$^{30}$ Une femme malade, touchée par la prédication des pères, demande le baptême, ce qui lui est accordé car « chacun jugeait qu'elle ne pouvait plus guère vivre ». Rien ne dit mieux que cette phrase la prudence des missionnaires. Cependant, une fois baptisée, cette femme recouvre la santé; se pose alors le problème de régler sa situation matrimoniale. Selon Yves d'Evreux, l'initiative vient de la néophyte elle-même:

mais un point la travaillait, savoir, qu'elle était femme d'un Tobajara, lequel avoit deux autres femmes, par ainsi elle ne pouvait vivre au mariage requis par les lois du Christianisme.

Yves d'Évreux ne précise pas, pour ses lecteurs, quelles sont les « lois du christianisme » requises pour le mariage, ils sont censés les connaître. Le cas de la femme tobajara est complexe, pour le missionnaire. La femme baptisée reste mariée avec son mari du temps de l'infidélité mais son mari ayant d'autres femmes, elle est de fait dans un mariage polygame. Or, une union polygame n'est pas acceptable pour l'Église.

Pour résoudre ce type de cas, les missionnaires ont recours à la règle canonique bien connue du «Privilège Paulin », ${ }^{31}$ qui est un des seuls moyens juridiques de rompre un mariage païen: "Nous remediasmes à cela, suivant le conseil de Saint Paul». Quand un converti ne peut plus vivre avec son conjoint infidèle qui l'empêche de vivre sa religion, Saint Paul $(1$ Cor. 7,16$)$ avait proposé de séparer le couple et de donner la possibilité au converti de se remarier. Ce privilège paulin est l'unique cas de dissolution (et non annulation) du mariage autorisé par l'Église. Ce principe canonique complexe est expliqué par les missionnaires français au mari tobajara, dont on imagine la perplexité:

Ainsi nous fisme dire à son mari que s'il voulait retenir cette sienne femme faite Chrétienne pour unique en se retirant des autres, qu'elle ne le quitterait point: mais s'il voulait la retenir comme auparavant en forme de concubine, que nous et les Grands des François lui permettions de le laisser, étant chose incompatible avec le Christianisme.

Le Privilège Paulin est ici modifié par le contexte politique. Saint Paul n'envisageait pas de forcer un infidèle à quoi que ce soit, les missionnaires, eux, obligent le mari non converti à faire un choix: soit il renonce à la femme chrétienne, soit il renonce aux autres. Pour une fois, Yves d'Évreux emploie le

30Yves d'Evreux, Voyage au Nord du Brésil, Paris, Édition F. Denis, 1864, Second traité, chapitre 2.

${ }^{31}$ G. Oesterlé, Privilège paulin, dans R. Naz (dir.), Dictionnaire de Droit canonique, Paris, Letouzey et Anet, 19351965, vol. VII, Paris, 1965, p. 230-280. 
discours de la contrainte en évoquant aux côtés des missionnaires ( nous ») les chefs temporels de la colonie («les Grands des Français »). L'affaire s'arrange, non sans une certaine forme de résistance de la part du mari:

le mari eut en ceci de la répugnance, néanmoins il s'y accorda à la fin, \& ainsi cette femme fut faite bonne chrétienne, demeurant seule femme avec lui.

On voit, par cet exemple, combien le mariage est un enjeu essentiel et délicat dans la relation missionnaire. Même si les missionnaires, comme Yves d'Évreux, admirent le fonctionnement de la famille indienne, la polygamie est une difficulté incontournable pour la « conversion » au christianisme. C'est parce qu'ils n'arrivent pas à résoudre les situations conjugales des Indiens au regard des normes chrétiennes que les missionnaires n'osent pas les baptiser et pour les Indiens, le mariage chrétien monogame et indissoluble est un obstacle pour adopter la croyance en Tupan dont on a vu qu'il était une figure attirante dans ce climat d'incertitudes et de dangers.

Dans le cas de cette femme tobajare, les missionnaires ont besoin de recourir à l'exception juridique (le privilège) et à la contrainte temporelle, pour régler sa situation matrimoniale. Du point de vue des missionnaires, il s'agit là d'un accident, en quelque sorte, car cette femme était censée mourir après avoir reçu le baptême. On comprend dès lors mieux, la prudence des missionnaires à baptiser des adultes. Cet épisode révèle, à la fois, la complexité des situations concrètes et la dimension conflictuelle du mariage, dans la relation missionnaire.

Pour les Indiens, l'abandon de la polygamie signifie une perte de statut social, de richesses économiques. Sur le plan symbolique aussi, ils sont dépossédés du sens de leurs unions, qui étaient liées à la mort des ennemis et à la vengeance. Le passage à la monogamie, dont on a vu que les missionnaires faisaient un préalable pour le baptême, est donc lourd de conséquences pour les Indiens. Yves d'Évreux évoque soit ouvertement, soit entre les lignes, la répugnance des Indiens pour ce nouveau type d'union. Le fait que la plupart des principaux préfèrent laisser leur fils aux mains des missionnaires, plutôt que se convertir eux mêmes, est sans doute une façon d'éviter « les lois du christianisme » concernant le mariage. Les fils, jeunes adultes, ne sont pas encore polygames, puisque la « saison de prendre femme » ne commence qu'à 25 ans.

Ainsi, loin d'être le récit monotone des progrès de l'évangélisation, ${ }^{32}$ les récits des pères capucins, Yves d'Évreux et Claude d'Abbeville racontent l'interaction missionnaire, un processus heurté et tâtonnant, où Indiens et missionnaires s'observent, se jaugent et discutent. Si les Indiens sont attirés par la figure toute puissante du Tupan des blancs, ils le sont moins par

\footnotetext{
32L'expression est empruntée à Hélène Clastres dans sa préface à l'édition de la Relation d' Yves d'Évreux [Voyage au Nord du Brésil fait en 1613 et 1614, présentation et notes d'Hélène Clastres, Paris, Payot, 1985] dans laquelle elle a enlevé une grosse partie du traité spirituel qu'elle juge inintéressant. J'ai analysé ce rapport ambigu des anthropologues avec les sources missionnaires dans l'article « De l'observation à la conversation: le savoir sur les Indiens du Brésil dans l'œuvre d'Yves d'Évreux », In: Charlotte de Castelnau-L'Estoile; MarieLucie Copete; Aliocha Maldavsky; Ines G. Zupanov, Missions d'évangélisation et Circulation des Savoirs XVIeXVIIle siècles, vol. 114, Madrid, Casa de Velazquez, Collection de la Casa de Velázquez, 2011, p. 269-294.
} 
la monogamie exigée par les pères. Le mariage est compris, par les uns et les autres, comme la pierre angulaire de la nouvelle société en train de se construire. Pour les missionnaires, il faut que les Indiens adoptent le mariage chrétien pour bâtir une nouvelle société chrétienne. Pour les Indiens, l'attention donnée aux blancs au mariage chrétien est le signe d'une dépossession et de l'entrée dans un nouveau monde que certains acceptent comme réponse aux incertitudes des temps nouveaux.

Les sources missionnaires ne sont pas seulement dépositaires d'un savoir observé par les missionnaires sur les Indiens, mais elles témoignent des échanges entre le missionnaire et les Indiens. Elles ne contiennent pas une description de la culture indienne d'origine, que les anthropologues pourraient atteindre en écartant les éléments missionnaires superflus; elles montrent cette culture en voie de transformation et d'adaptation. Il serait vain de vouloir démêler, dans ces conversations, ce qui serait authentiquement indien ou ce qui serait purement missionnaire. Les traces de ces interactions nous renseignent sur les deux acteurs en présence, Indiens et missionnaires, leurs stratégies mutuelles de reconnaissance, d'appropriation et de rejet, sur fond de rapports de force changeants. 